Luigi BISIO

\title{
Note corologiche, tassonomiche ed ecologiche su Pterostichus (Pterostichus) honnoratii (Dejean, 1828) (Coleoptera Carabidae)
}

\begin{abstract}
Riassunto: Viene pubblicata un'analisi della corologia di Pterostichus (Pterostichus) honnoratii (Dejean, 1828) nelle Alpi Occidentali. La sottospecie sellae (Stierlin, 1881) è nota delle Alpi Liguri, Marittime e Cozie meridionali. La sottospecie nominale occupa le Alpi Cozie settentrionali, le Graie, le Pennine e, più a Ovest, le Prealpi francesi di Vaucluse (Mont Ventoux) e le Prealpi del Delfinato (Vercors). La sottospecie ludovici Schatzmayr, 1930 è nota delle Alpi svizzere, sui monti intorno al Lago di Ginevra. Inoltre, sono riassunti i caratteri esterni che permettono di distinguere le tre sottospecie. Esse differiscono per la morfologia degli angoli posteriori (ssp. sellae: ottusi e smussati; ssp. honnoratii: retti e sporgenti) e degli angoli anteriori (honnoratii: sporgenti; ludovici: poco sporgenti) del pronoto. Infine, sono proposte alcune osservazioni sull'ecologia di questa specie. Lungo il versante italiano delle Alpi Occidentali, $P$. honnoratii vive esclusivamente negli orizzonti alpino e altoalpino dove popola prevalentemente i ghiaioni calcarei e sfasciumi di calcescisti ai margini dei nevai. In territorio francese, si rinviene anche a quote inferiori in foresta.
\end{abstract}

Abstract: Taxonomical, chorological and ecological notes about Pterostichus (Pterostichus) honnoratii (Dejean, 1828) (Coleoptera Carabidae) The distribution of Pterostichus (Pterostichus) honnoratii (Dejean, 1828) in Western Alps is reviewed. The subspecies sellae (Stierlin, 1881) is known as endemic of the Ligurian Alps, of the Sea Alps and of the southern Cottian Alps. The nominal subspecies lives in the northern Cottian Alps, in the Graian Alps, in the Penninian Alps and, further West, in the French Prealps of Vaucluse (Mont Ventoux) and of the Dauphiné (Vercors). The subspecies ludovici Schatzmayr, 1930 is known of the Swiss Alps, at the mountains surrounding Lake Geneva. Moreover, the external traits that allow to distinguish the three subspecies are summarized. They differ in the morphology of posterior angles (ssp. sellae: obtuse and blunt; ssp. honnoratii: right and protruding) and anterior angles (ssp. honnoratii: protruding; ssp. ludovici: little protruding) of pronotum. Furthermore, some ecological remarks about this species are presented. Along the Italian slope of The Western Alps, $P$. honnoratii lives in the alpine and high-alpine areas where it predominantly inhabits the limestone screes and the debris of calcareous schists at the edges of the snowfields. In France, it is found also at a lower altitude in forest.

Key words: Coleoptera, Carabidae, Pterostichus, Western Alps, distributions, taxonomy, ecology.

\section{INTRODUZIONE}

Con la presente nota lo scrivente, che già in passato si è occupato di Pterostichus (Pterostichus) honnoratii (Dejean, 1828) in un lavoro dedicato ad alcuni Pterostichus orofili del Piemonte (Bisio, 1995), intende riassumere le conoscenze a tutt'oggi disponibili sulla corologia, sulla tassonomia e sull'ecologia di questa interessante specie, endemita delle Alpi Occidentali (corotipo: W-Alpino, sensu Vigna Taglianti, 2005). È da precisare che il territorio delle Alpi Occidentali viene qui considerato - secondo l'attuale suddivisione SOIUSA - in senso lato, cioè esteso ad Ovest alle Prealpi di Vaucluse (Mont Ventoux) e alle Prealpi del Delfinato (Vercors), rilievi alquanto distanti dalla cresta assiale.

\section{Catalogo Topografico}

P. honnoratii è nota delle seguenti stazioni (Fig. 1) [Per ogni località riporto la citazione bibliografica, il raccoglitore e chi me l'ha segnalata, se di- verso dal raccoglitore (AVT=Augusto Vigna Taglianti; $\mathrm{AC}=$ Achille Casale; $\mathrm{GA}=$ Gianni Allegro; $\mathrm{LB}=$ Luigi Bisio; $\mathrm{PMG}=$ Pier Mauro Giachino; $\mathrm{RP}=$ René Pupier); $\mathrm{i}$ dati desunti dal sito dedicato alle biodiversità dei Parchi del Mercantour e delle Alpi Marittime (AA.VV., 2007) vengono indicati con l'acronimo ATBI]:

ssp. sellae (Stierlin, 1881)

Francia: Alpes-Maritimes (Provence-AlpesCôte-d'Azur): Alpes de Lantosque (Sainte-Claire Deville, 1902); Col du Ciavalet (Sainte-Claire Deville, 1902); Col de la Colombière (Sainte-Claire Deville, 1902); Col de Champs (Sainte-Claire Deville, 1902); Monte Mounier (Sainte-Claire Deville, 1902; Magistretti, 1965); Massif du Mont Mounier, 15.VI.1958, Bonadona leg. (RP); Vallon de Jallorgues, 20.06.1946, Bonadona leg. (RP); Parc National du Mercantour, Col de la Bonnette (Saint-Dalmas-le-Selvage), 19.VII.196210.IX.1962, Cavalier leg. (dati ATBI); Parc National du Mercantour, Col du Restefond (Jausiers), 9.VII.19668.VIII.1966, Cavalier leg. (dati ATBI); Parc National 
du Mercantour, Saint-Étienne-de-Tinée, gare supérieure du téléférique de Las Donas (Auron) m 2275, 13.VI.2002, Cornet leg. (dati ATBI); Saint-Étienne-deTinée (Sainte-Claire Deville, 1902; Magistretti, 1965). Alpes-de-Haute-Provence (Provence-Alpes-Côted'Azur): ravin de Méouilles (Allos) m 1000, 2 es. IX.1962 (RP); Cime de Valcibière (Sainte-Claire Deville, 1902); Montagne de Valcibière (Allos) m 2375, Villard, leg. (RP); Col d'Allos (Sainte-Claire Deville, 1902); Col d'Allos (versant S) (Allos) m 2247, VII.1927, P. Joffre leg. (RP); Col d'Allos (versant S) (Allos) m 2247, 14.VI.1964-21.VI.1959, Bonadona leg. (RP); Col de la Petite Cayolle (versant S) (Allos) m 2639, IX.1962 (RP); Lac d'Allos (Allos) m 2220, Villard leg. (RP); Montagne de Blayeul (Sainte-Claire Deville, 1902); Montagne de Blayeul (versant E) (Beaujeu) m 2180, Peyerimhoff leg. (RP); Peyresq, Cabane Vieille (Colmars) m 2100, 1 es. 15.VII.1985, 2 es. 14.07.1986, Bérard leg., (RP); Col des Champs (Colmars) m 2100, 26.VII.1985, Bonadona leg. (RP); Col des Champs (Colmars) m 2100, 27.VIII.200031.VIII.2000 (RP); Col de l'Enombrette (versant sud) (Colmars), m 2400, 8.VIII.1998, (RP); Lacs des Lignin (Colmars) m 2273, 17.VI.2003, Coache leg. (RP); versant NO de la Tête des Blaves (Larche), m 2200, 1 es. 14.VII.1999 (RP); Vallon de l'Oronaye (Larche) m 2200-2400, 14.VII.1999 (RP); ravin de l'Elve (Méolans-Revel), 3 es. 15.VIII.1997 (RP); ravin de Léoure (Méolans-Revel), 2 es. 15.VIII.1997, R. (RP); ravin des Lausas (Méolans-Revel), m 1950, 31.V.1997, Coache leg. (RP); Cabane Vieille (Thorame-Haute), m 2049, 14.07.1985, Bérard leg. (RP); Grand Coyer (sommet) (Thorame-Haute), m 2693, 8.VII.2005, Coache leg. (RP); le Petit Coyer (sommet) (Thorame-Haute), m 2580, 5.VII.2005, Coache leg. (RP); Plan du Rieu (Thorame-Haute), $\mathrm{m}$ 2075, 7.VII.2005, Coache leg. (RP); le Signal (Uvernet-Fours) m 2310, 3 es. 2.VI.2007 (RP); Col d'Allos (versant nord) (Uvernet-Fours), m 2247, Peyerimhoff leg. (RP); montée au col d'Allos (Uvernet-Fours) m 2100, 1 es. 24.VII.1973, Cantot leg. (RP).

Italia: Piemonte CN: Val Pesio: "Val Pesio" (Stierlin, 1881); Monte Marguareis, m 2000, 1 es. 5.VII.1941, B. Bari leg. (PMG); Val Vermenagna: Lago degli Alberghi (Palanfré) m 2000, 1 es.12.VI.1988 (Bisio, 1995); Valle Stura di Demonte: Monte Pebrun (dint.Colle Puriac) m 2500, 3 es. 12.VIII.1991, Vigna Taglianti leg. (Bisio, 1995); Vallone Puriac m 1850-2150, 1 es. 8.VII.1987, Vigna Taglianti leg. (Bisio, 1995); Bassa di Colombart m 2400,

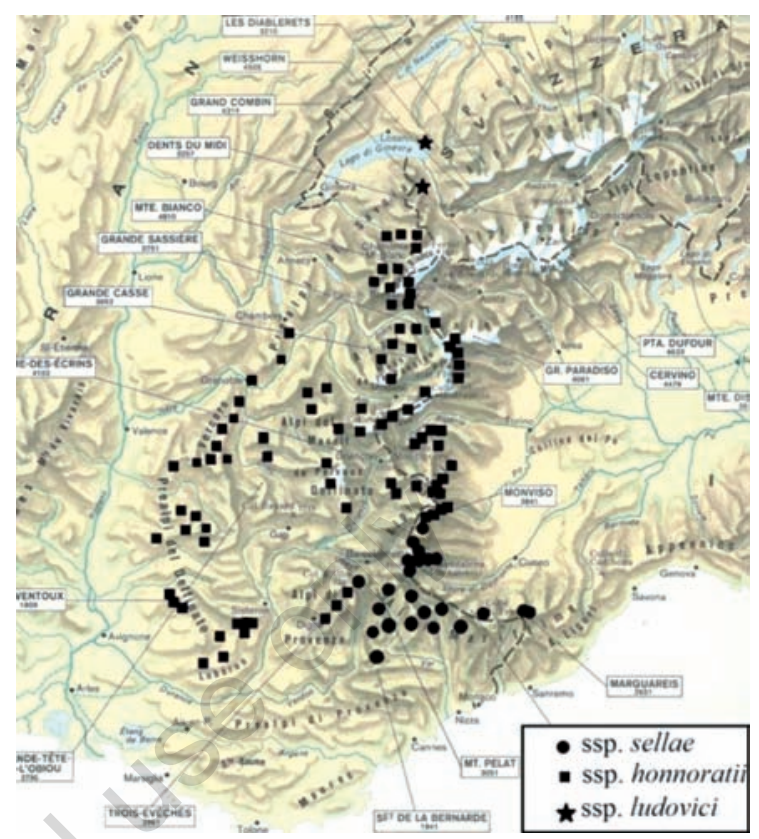

Fig. 1. Distribuzione geografica di Pterostichus honnoratii (cfr. anche Coulon et al., 2000).

2 es. 2.IX.1996 (AVT); Colle della Maddalena m 2000, 1 es. 24.VII.1966, Osella leg. (Bisio, 1995); Colle della Maddalena m 2000, Casale leg. (Bisio, 1995); Vallone Oronaye m 2200-2300, 1 es. 30.VI.1977, Vigna Taglianti leg. (Bisio, 1995); Laghi di Roburent (Argentera) m 2400, 4 es. 9.VI.1985 (Bisio, 1995); Rocca La Meja (parete S) m 2600, 1 es. 20.VII.1987, Vigna Taglianti leg. (Bisio, 1995); Vallone della Madonna (Sambuco) m 1300 (esemplare probabilmente fluitato), Sciaky leg. (Bisio, 1995); Cima Ruissas, (Colle Valcavera) m 2500, 1 es. 9.VI.1998 (AVT); Colle di Valcavera m 2300, 2 es. 29.V.1983, 1 es. 30.V.1990, Giuntelli leg. (Bisio, 1995); Colle Valcavera m 2400, 2 es. 16.VI.1999, Vigna Taglianti \& Mei leg. (AVT); Colle del Mulo m 2500, 1 es. 2.VIII.1999, Osella leg. (AVT); Cima Omo m 2000 (Schatzmayr, 1930); Cima Omo, 1 es. 14.VI.1986, Giuntelli leg. (Bisio, 1995); Val Maira: Prato Gelato (Acceglio) m 2100, 1 es. 30.VI.1968, B. Bari leg. (PMG); Passo Gardetta m 2400, 1 es. 25.VII.1974, Vigna Taglianti leg. (Bisio, 1995); Passo di Rocca Brancia m 2400, 1 es. 21.VI.1987 (Bisio, 1995); Passo Escalon m 2400, 1 es. 15.VI.2002, Alle- 
gro leg. (Bisio \& Giuntelli, 2011); Colle del Sautron (Saretto) m 2500, 1 es. 2.VII.1986, 1 es. 30.VI.1994 (Bisio, 1995); La Colletta m 2600, 1 es. 29.VI.2002, Allegro leg. (Bisio \& Giuntelli, 2011).

Forme di transizione tra la ssp. nominale e la ssp. sellae Francia: Alpes-de-Haute-Provence (Provence-Alpes-Côte-d'Azur): Montagne du Cheval Blanc (versant E) (Thorame-Basse) m 2000, 4 es. 29.VI.1996, (RP); Montagne du Cheval Blanc (sommet) (Thorame-Basse) m 2323, 29.VI.19969.IX.2001-21.VI.2003, Coache leg. (RP); montagne de Boules (versant E) (Thorame-Basse) m 2391, 7.VII.2002, Perez leg. (RP); montagne de Tournon (Thorame-Basse) m 2243, 15.IX.2002, Perez leg. (RP); Mourre-Frey m 2291 (Thorame-Basse) 19.VI.2003, Coache leg. (RP); ravin de Raichas (Thorame-Basse) m 1877, 15.VI.2003, Coache leg. (RP).

Italia: Piemonte CN: Val Maira:Vallone di Traversiera (Acceglio) m 2000, 1 es. 15.V.1983 (Bisio, 1995: sub honnoratii sellae). Val Varaita: Pian Traversagn m 2200 (Schatzmayr, 1930: 282); Colle di Saint-Veran (Chianale) m 2500, 2 es. 16.VIII.1989 (Bisio, 1995: sub honnoratii honnoratii).

ssp. honnoratii (Dejean, 1828)

Francia: Vaucluse (Provence-Alpes-Côted'Azur), Mont Ventoux: Mont Ventoux (Jeannel, 1942); Mont Ventoux, de Boisys leg. (RP); Mont Ventoux, 13.VI.1959, Bonadona leg. (RP); Mont Ventoux, 1 es. 30.V.1982, Colomb leg (RP); Mont Ventoux (versante S) m 1400-1500, 8 es. 1.V.2008-30.V.2008 (pitfall traps) (AC); ibidem (versante N) m 1400, 7 es. 21.VII.2011, A. \& G. Casale leg. (AC); route de Malaucène (Beaumont-de-Ventoux), m 1300, IX.1962, IX.1966 (RP); Col des Tempêtes (Bédoin) m 1841,1 es. 3.IX.2003 (RP). Alpes-de-Haute-Provence (Provence-Alpes-Côte-d'Azur), Montagne de Lure: Montagne de Lure, 1 es. 31.V.1982, Colomb leg. (RP); Saint-Etienne-les-Orgues, combe de Chavalet, $\mathrm{m}$ 1700, 2 es. IX.1961, 1 es. VII.1966 (RP); Saint-Etienne-lesOrgues, ouest de Morteiron, m 1680, 3 es. 14.VI.2002 (RP); Saint-Etienne-les-Orgues, combe de Glacières $\mathrm{m}$ 1680, 14.VI.2002 (RP); Saint-Etienne-les-Orgues, pylones de Lure $\mathrm{m}$ 1820, 8.V.1996-4.VI.2001-24.V.200214.IX.2002, Coache leg. (RP); Saint-Etienne-lesOrgues, signal de Lure, m 1826, 20.VI.1965, Dufay leg. (RP); Saint-Etienne-les-Orgues, signal de Lure, $\mathrm{m}$ 1826, 24.V.2001-4.VI.2001, Coache leg. (RP); Saint-
Etienne-les-Orgues, sommet de Morteiron m 1708, 14.IX.2002, Perez leg. (RP); Saint-Etienne-les-Orgues, station de Lure m 1590, 31.IV.1989 Coache leg. (RP); Pas de la Croix (Cruis) m 1502, 24.V.2001, Coache leg. (RP); Pas de la Graille (Cruis) m 1597, 20.V.19957.VI.2001, Coache leg. (RP); Pré de Fayard (Lardiers) m 1500, 30.IV.2002, Coache leg. (RP); la Fayée (Noyers-sur-Jabron) m 1500, 8.VII.1995-27.VII.1997, Coache leg. (RP). Alpes-de-Haute-Provence (Provence-Alpes-Côte-d'Azur), Massif du Cheval Blanc: Montagne de Carton (Prads-Haute-Bléone) $\mathrm{m}$ 1750, 1 es.16.IX.2001 (RP); bois de la Baisse (PradsHaute-Bléone) m 1350-1550, 20.VI.1998, Vaucel leg. (RP); Faillefeu (Prads-Haute-Bléone) m 1340, Bedel leg. (RP); le Talon (Prads-Haute-Bléone) m 1991, 19.VI.2005, Coache leg. (RP). Alpes-de-HauteProvence (Provence-Alpes-Côte-d'Azur), Massif des Monges: les Monges (sommet) (Authon) $\mathrm{m} 2115$, 14.VII.1994, 6.VII.1996, Coache leg. (RP); les Monges (le Grand Bois) (Bayons) m 1600, 17.IX.1994 Richoux leg. (RP); les Monges (versant nord) (Bayons) m 1850, 17.IX.1994, Richoux leg. (RP); Montagne de Blayeul (versant E), (Beaujeu), m 2180, Peyerimhoff leg. (RP). Alpes-de-Haute-Provence (ProvenceAlpes-Côte-d'Azur): la Colle (Céreste, Luberon), m 856, VI.2000, 9.VII.2000, 14.VIII.2000, Coache leg. (RP); les Dourbes (Digne-les-Bains) m 1060, Caillol leg. (RP); Villard de Lans (Jausiers), m 1475, Rizaucourt leg. (RP); aven de Néoures (la Rochegiron), $m$ 1386, 1.VII.2005, Coache leg. (RP); les Brieux (Redortiers), m 910, 16.IX.2004, Coache leg. (RP); Valbelle, $m$ 550, 27.VII.1974, Voisin leg. (RP). Hautes-Alpes (Provence-Alpes-Côte-d'Azur): col du Lautaret, m 2058, 1 es. 7.V.1992, Colomb leg. (RP); Orcières, de Boissy leg. (RP); Château-Queyras, de Boissy leg. (RP); Col de la Fraissinière $\mathrm{m}$ 2700, de Boissy leg. (RP); col d'Izoard, m 2500, 24.VI.1994, Teissier leg. (RP); Ristolas m 1900, 27.VI.1994, Teissier leg. (RP). Drôme (Rhône-Alpes) Vercors: forêt de Lente $\mathrm{m} \mathrm{1100,1}$ es. V.1961, Berne leg. (RP); Col de Lachau (Vassieux-en-Vercors) m 1330, 1 es. V.1967 (RP);. Col du Rousset (Chamaloc) m 1250, 3 es. 16.IX.1969 (RP); Drôme (Rhône-Alpes) Diois: forêt de Saou (piéges) m 500, VI.1979, Bonadona leg. (RP); forêt de Saou (pièges), m 500, 3 es. 9.IX.1977 (RP); Serre de Montfort (la Chaudière) $\mathrm{m} \mathrm{1000,7}$ es. 13.IV.1981 (RP); les Coulasses (Aucelon) (in Faggeta) m 750, 1 es. 25.VI.1989 (RP). Isère (Rhône-Alpes): Grenoble, Sinigaglia leg. (Schatzmayr, 1930); Route 
d'Allevard au Galibier, 1 es. 22.VII.1973, Cantot leg. (RP); Source du Guiers Vif, 1 es. 3.VII.50, H. Henrot leg. (PMG); Savoie (Rhône-Alpes): Mont Cenis, 20.VI.1972, Bonadona leg. (RP); Col du Petit-SaintBernard, m 2100, 6 es. 19.VIII.2010 (AVT); ibidem, 4 es. stessa data (AC). Haute-Savoie (Rhône-Alpes): Mont Joly (Sainte-Claire Deville, 1902); Pralognan, 18.VII.1922, Hardy leg. (RP). A proposito della "Région Rhône-Alpes", occorre rilevare che nel lavoro di Coulon et al. (2000) dedicato a tale territorio è riportata una cartina di distribuzione della specie dalla quale si evince la sua ampia diffusione nella regione. Peraltro, poiché le oltre sessanta stazioni note non sono citate nel testo, non è possibile riportarle nel presente lavoro. Esse riguardano i seguenti "districts naturels": Chablais, Bornes, Mont Blanc-Beaufortin, Vanoise, Chartreuse-Mont du Chat, Maurienne-Grandes Rousses, Oisans, Dévoluy-Trièves-Matheysine, Vercors, Diois, Tricastin e Baronnies-Ventoux.

Italia: Piemonte, CN: Val Varaita: Vallone dell'Agnello m 2400, 1 es. 8.VII.1974, Vigna Taglianti leg. (Bisio, 1995); Colle dell'Agnello, 2 es. 5.VII.1997, Diotti leg. (Bisio \& Giuntelli, 2008); ibidem, 1 es. 8.VIII.1992, 1 es. 18.VII.1994, Bosi leg. (GA) (Bisio \& Giuntelli, 2008); Vallone di Soustra (Chianale) $m$ 2000, 1 es. 24.V.1980, Giuntelli leg. (Bisio, 1995); ibidem a m 2100, 2 es. 25.V.1998 (Bisio \& Giuntelli, 2008); Chianale $\mathrm{m} 1850,2$ es. 17.VI.1968, Vigna Taglianti leg. (Bisio, 1995). Valle Po: Pian Giasset m 2200-2250, Cavazzuti leg. (Bisio, 2010); Monte Ghincia Pastour m 2400, Cavazzuti leg. (Bisio, 2010); Lago Fiorenza m 2010-2130, Cavazzuti leg. (Bisio, 2010). Piemonte, TO: Val Pellice: Colle della Gianna (Comba dei Carbonieri, Bobbio Pellice), 1 es. 1.VII.1997 (GA); Col Barant (Comba dei Carbonieri, Bobbio Pellice) $\mathrm{m}$ 2300, 1 es. 27.VI.1977, 2 es. 26.VI.1978 (Bisio, 1995); ibidem, 6 es. 2.VII.1997 (GA); Rifugio Granero (Conca del Prà, Bobbio Pellice) m 2350, 1 es. 6.VII.1978 (Bisio, 1995); Colle dell'Urina (Conca del Prà, Bobbio Pellice) m 2300-2500, pl. es. 2.VII.1976, 2.VII.1978, 28.VI.1980, 21.VI.1981 (Bisio, 1995); ibidem 2 es 19.VIII.2002 (LB); Col Boucìe (Villanova, Bobbio Pellice) $\mathrm{m}$ 2600, 2 es. 15.VII.1979 (Bisio, 1995); Alpe Bancet (Villanova, Bobbio Pellice) m 2250, 3 es. 15.VI.1997 (Bisio, 2004); Colle Giulian (Bobbio Pellice) $\mathrm{m}$ 2400, 1 es. 9.VII.1978 (Bisio, 1995). Val Germanasca: Tredici Laghi (Magistretti, 1968); Colle del Beth (Massello) m 2700, 1 es. 20.VIII.1978 (Bisio, 1995); Pian delle Sagne (Vallone del Pis, Massello) m
2200, 1 es. 22.VIII.2004 (Bisio, 2007). Val Chisone: Fenestrelle m 2000, 8 es. 7.VIII.1961, Papini leg. (AVT); Vallone dell'Albergian, 1 es. 1908, Ganglbauer leg. (PMG); Laghi dell'Albergian m 2350 (Schatzmayr, 1930); ibidem a m 2550, 1 es. 18.VIII.1997 (LB); Colle dell'Albergian m 2700, 1 es. 16.VII.1978 (Bisio, 1995); Monte Albergian m 2300, 2 es. 26.VI.1986 (Bisio, 1995); Clot della Soma (Pragelato) m 2200, Casale leg. (Bisio, 1995); Pian Soldà (Val Troncea, Pragelato) $\mathrm{m}$ 2300-2470, 1 es 19.VI.2003, Cavazzuti leg. (GA); ibidem m 2300-2600, 1 es.16.VII.2003, Cavazzuti leg. (GA); Monte Alpette (Sestrière) $\mathrm{m}$ 2200, 4 es. 30.VI.1983, P.M. Giachino leg. (Bisio, 1995); Colle del Sestrière m 2021 (Schatzmayr, 1930); Monte Fraiteve (Sestrière) m 2200, 3 es. 1.VII.1983, P.M. Giachino leg. (Bisio, 1995); Col Basset (Sestrière) m 2300-2400, pl. es. 9.VI.1979, 1.VI.1980 (Bisio, 1995); ibidem pl. es. 19.VI.2008, Bisio leg.; Monte Assietta m 2550, 1 es. 31.VIII.1978 (Bisio, 1995); Colle dell'Assietta m 2300 (Schatzmayr, 1930); ibidem m 2200, 1 es. 26.VIII.1971 (AVT); Colle delle Finestre (Usseaux), 2 es. 11.VIII.1968, Cantamessa leg. (Allegro, 2000); Monte Pelvo (Fenestrelle) m 2100, 5 es. 26.VI.1991 (Bisio, 1995); Prà Catinat-Colle dell'Orsiera m 1600-2600, pl. es. in trappole 2007-2008 (Allegro \& Viterbi, 2010); Punta Cristalliera versante SW (Villaretto) m 24002500, 23.VIII.2010 (LB). Val Sangone: Coazze (Sainte-Claire Deville, 1902); Coazze, Dodero leg. (Schatzmayr, 1930). Valle di Susa: Colle delle Finestre m 2215 (Schatzmayr, 1930); ibidem a m 2000-2100, pl. es. 19.VI.1977, 18.VI.1978 (Bisio, 1995); ibidem a m 2100, 13 es. 26.VIII.1971, 11 es. 27.VI.1997, 10 es. 28. VI.1997 (AVT); ibidem a m 2176, 5 es. 29.VI.1983 (PMG); ibidem, 1 es. 3.VII.1994 (GA); Cesana Torinese (Casale et al., 2006); Bardonecchia (Magistretti, 1965); Rifugio Scarfiotti (Vallone di Rochemolles, Bardonecchia) m 2100, 3 es. 27.VI.1981 (Bisio, 1995); Pian dei Morti (Vallone Sommellier, Bardonecchia) $\mathrm{m}$ 2500, 1 es. 17.VII.2010 (LB); Monte Niblé (Vallone Galambra, Exilles) m 1900, pl. es. 4.VIII.1996, 6.VI.1997 (LB); Moncenisio (Schatzmayr, 1930; Casale et al., 2006); ibidem 2 es. 24.V.1986, Giuntelli leg. (Bisio, 1995). Valli di Lanzo: Lago Malciaussia (Usseglio) m 1820, 1 es. 11.V.1980, 1 es. 16.VI.1989, Giuntelli leg. (Bisio, 1995); Pian della Mussa m 1845, 1 es. 26.VII.1978, 2 es. 14.VII.1979, Giuntelli leg. (Bisio, 1995); Balme (Magistretti, 1965); ibidem a m 2128 (Casale et al., 2006: erroneamente indicata in provincia di Aosta). Valle Orco: Monte Levanna (Sainte- 
Claire Deville, 1902; Schatzmayr, 1930); Lago Serru, m 2300, 1 es. 6.VII.1953, B. Bari leg. (PMG); Colle del Nivolet m 2450 (Casale et al., 2006); Costa della Civetta (Punta Violetta) m 2100, 1 es. 9. VI.1991 (Bisio, 1995). Valle d'Aosta, AO: Valsavarenche: Valsavarenche, Dodero leg. (Schatzmayr, 1930); Valgrisenche: Rifugio Bezzi (2 km a S, verso il Ghiacciaio Vaudet) $\mathrm{m}$ 2350, 2 es. 3.VIII.2008 (Götz com. pers., 2012); Valle di La Thuile: Val Chavannes, 2 es. VI.1966, leg. ?, ex collezione Casiccia (G. Franzini, com pers., 2011); Val Veny: Lago Combal (Courmayeur) m 2000, 1 es. VII.1948, Pozzi leg. (PMG); Ghiacciaio delle Stellette m 2600, L. Giachino leg. (Casale \& Vigna Taglianti, 1993).

ssp. ludovici Schatzmayr, 1930

Svizzera: Rochers de Naye (Lousanne) $\mathrm{m}$ 2045 (Schatzmayr, 1930); Col de Bretolet (Colombes), 1 es. 20.IX.1963 (AVT); ibidem, 1 es. 25.VIII.1964, Sbordoni leg. (AVT).

Lungo il versante francese, quindi, l'areale della specie, già delineato sommariamente da SainteClaire Deville (1902) e in modo più dettagliato da Jeannel (1942), si estende dal Lago di Ginevra alle Alpi Marittime e comprende, più a Ovest, le Prealpi di Vaucluse (Mont Ventoux) e le Prealpi del Delfinato (Vercors).

Lungo quello piemontese, la specie è invece diffusa dalle Alpi Liguri alle Graie, tra i seguenti limiti attualmente conosciuti:

- la Val Pesio a Sud;

- la sinistra orografica dell'alta Valle Orco a Nord (contrafforti occidentali del Gran Paradiso).

Occorre peraltro rilevare che, a Nord della Val di Susa - in particolare nelle Valli di Lanzo dove non mancano substrati carbonatici che, almeno in teoria, sembrerebbero molto favorevoli alla specie (v. Ecologia) - le sue popolazioni sembrano avere una diffusione decisamente più discontinua, come dimostra il numero estremamente ridotto di reperti ottenuti in tale territorio.

Per quanto concerne invece la Valle d'Aosta, di questa valle sono note soltanto cinque stazioni, probabile esito di tracimazioni da territori confinanti. Nel caso della Valsavarenche, tale ipotesi è alquanto probabile data la presenza della specie al Colle del Nivolet che si trova in corrispondenza della testata della valle. Per quanto riguarda invece le quattro stazioni rispettivamente della Valgrisenche (1), della Valle di La Thuile (1) e della Val Veny (2), la loro posizione alquanto marginale, immediatamente a ridosso del confine con la Francia, fa ritenere (cfr. Casale \& Vigna Taglianti, 1993; Allegro et al., 2011) che si tratti di popolamenti transalpini. Depone a favore di tale ipotesi l'ampia soluzione di continuità tra queste tre popolazioni e quelle piemontesi. La specie, infatti, risulta assente nel resto della Valle d'Aosta (cfr. Focarile, 1974, 1987; Allegro \& Chiarabaglio, 2008; Allegro \& Viterbi, 2009) e nelle valli canavesane nord-orientali (cfr. Bisio, 2003, 2012).

\section{CONSIDERAZIONI TASSONOMICHE}

Pterostichus honnoratii appartiene (sensu Coulon et al., 2011) al gruppo hagenbachii formato - oltre che dalla specie in oggetto - da Pterostichus (Pterostichus) hagenbachii (Sturm, 1824) e da Pterostichus (Pterostichus) devillei (Puel, 1924). Dopo la sua descrizione su esemplari provenienti dalle Basses Alpes (attualmente denominate Alpes-de-Haute-Provence) da parte di Dejean (1828), Stierlin (1881) descrisse, su due esemplari della Val Pesio, Pterostichus sellae come specie distinta. Sainte-Claire Deville (1902), invece, considerò il taxon una "varietas" meridionale di $P$. honnoratii, rango che venne successivamente confermato anche da Caillol (1908), da Porta (1923) e da Winkler (1924-1932). Schatzmayr (1930, 1942-1943) rilevò differenze morfologiche importanti nelle popolazioni più settentrionali che occupano, in territorio svizzero, i monti intorno al Lago di Ginevra e le denominò ssp. ludovici. Articolò, quindi, la specie in oggetto in tre sottospecie delle quali fornì nei dettagli la descrizione dei caratteri diagnostici che consentono di distinguerle. Essi sono così riassumibili:

- lati del pronoto appena sinuati davanti agli angoli posteriori che sono nettamente ottusi e smussati (ssp. sellae) (Fig. 2);

- lati del pronoto fortemente sinuati verso la base, angoli posteriori quasi retti e sporgenti (ssp. honnoratii) (Fig. 3);

- lati del pronoto debolmente sinuati davanti agli angoli posteriori; angoli anteriori poco sporgenti (ssp. ludovici).

Il rango subspecifico di sellae è stato successivamente mantenuto da tutti gli autori francesi e italiani (Jeannel, 1942; Magistretti, 1965; Casale \& Vigna Taglianti, 1993; Vigna Taglianti, 1993, 2005; 


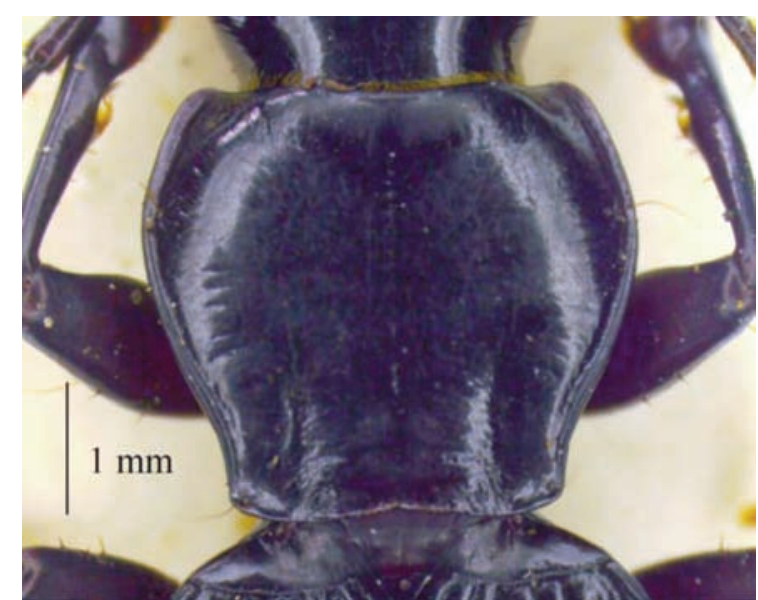

Fig. 2. Pronoto di P. honnoratii sellae del Passo Escalon (fotografia di Gianni Allegro).

Bisio, 1995), che si sono occupati di $P$. honnoratii in lavori specifici, cataloghi o checklists riguardanti i Carabidi del proprio territorio nazionale. Ovviamente, trattandosi di popolazioni marginali, estranee sia alla fauna francese, sia a quella italiana, nessuno di questi autori ha preso in considerazione la questione della validità della ssp. ludovici, validità che è stata poi mantenuta in tempi più recenti da Löbl \& Smetana (2003), da Vigna Taglianti (2004) e da Lorenz (2005).

Recentemente, Pupier (2007) ha esaminato i due esemplari femmina che costituiscono la serie tipica della ssp sellae, designandone il lectotypus. Confrontando, poi, il pronoto di questi due esemplari con quello di individui provenienti dalla località tipica della sottospecie nominale (più precisamente dalla Montagne de Lure), ne ha ribadito le nette differenze morfologiche già evidenziate dagli autori precedenti. Tuttavia l'autore stesso, dopo aver esaminato l'abbondante materiale a disposizione proveniente da pressoché tutto l'areale francese della specie, ha anche evidenziato che tali differenze tendono ad attenuarsi a mano a mano che ci si allontana dalle località tipiche, tanto che in alcune zone l'attribuzione degli esemplari ad una o all'altra sottospecie è alquanto aleatoria. È il caso, ad esempio, della Montagne du Cheval-Blanc, dove (Pupier, 2007; Coulon et al., 2011) si trovano individui dalla morfologia alquanto variabile che li rende difficilmente classificabili. L'au-

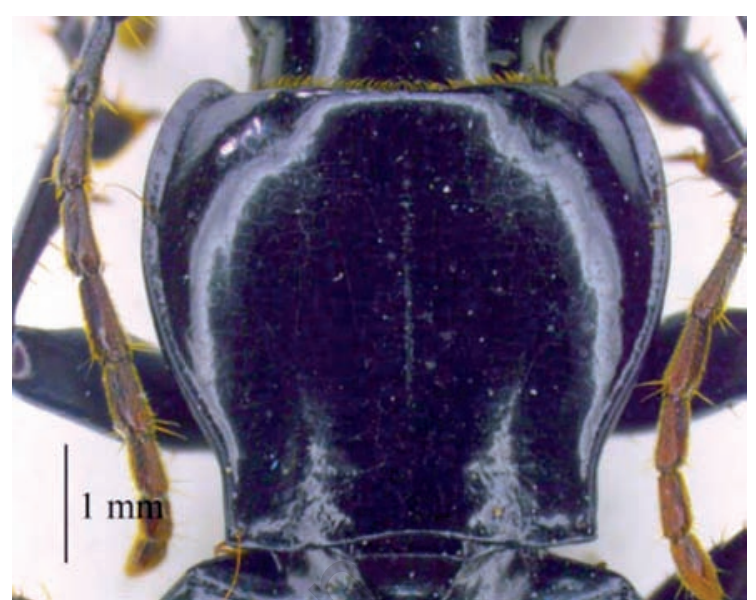

Fig. 3. Pronoto di P. honnoratii honnoratii del Col Barant (fotografia di Gianni Allegro).

tore stesso (Pupier com. pers., 2012) mi comunica che la sinuosità del pronoto, di fatto assente nella serie tipica della Val Pesio e negli individui del Mont Mounier, tende ad accentuarsi gradualmente a mano a mano che ci si sposta verso Nord e diviene netta nei pressi del Col d'Allos e nei dintorni di Larche. Pertanto, nelle popolazioni del versante francese non sembra esistere una netta separazione geografica tra la due forme, quanto piuttosto una variabilità clinale. Egli perciò conclude: "Il ressort de cette analyse que le maintien comme sous-espèce de la forme sellae est assez artificiel; il existe une différence morphologique appréciable entre les deux formes, mais la variabilité dans la zone de contact est telle que l'on ne peut parler d'une séparation géographique. On se trouve là dans un cas où les règles de la systématique sont difficilement applicables. C'est donc par pure convention que je conserve le statut de sous-espèce à la forme sellae". Queste ultime considerazioni portano poi lo stesso Pupier a confermare il rango subspecifico di sellae anche nel recente aggiornamento della "Faune de France" (Coulon et al., 2011) del quale ha curato la parte dedicata ai Pterostichini.

Per quanto concerne le popolazioni italiane, il limitato numero di esemplari provenienti dalla parte meridionale dell' areale (dove essa, in apparenza, è più sporadica) consente uno studio meno approfondito e, forse, meno oggettivo della variabilità morfologica 
della specie rispetto alle popolazioni d'oltralpe. Tuttavia, non si può non rilevare che il quadro che ne emerge pare confermare pienamente la validità subspecifica di sellae. Sono sicuramente da ascrivere a quest'ultimo taxon, infatti, gli esemplari esaminati provenienti dalla zona compresa tra la Val Vermenagna (Lago degli Alberghi) e la destra orografica della Val Maira (Passo Escalon e Col Sautron): in essi non sono state rilevate né differenze morfologiche al variare della valle di provenienza, né conseguentemente un'eventuale variabilità in senso clinale. Solo a partire dal versante opposto di quest'ultima valle iniziano a comparire - come già evidenziato da Schatzmayr (1930: 282) a proposito degli esemplari del Pian Traversagn in Val Varaita - individui (ad esempio, quelli provenienti dal Vallone di Traversiera in Val Maira e dal Colle di Saint-Veran in Val Varaita) che, presentando già una sinuosità leggermente più accentuata nel pronoto e angoli posteriori meno ottusi e più sporgenti, paiono a tutti gli effetti delle forme di transizione. Presentano, invece, in modo netto la morfologia propria della ssp. nominale gli esemplari della Val Varaita provenienti dal Colle dell'Agnello (Allegro, com. pers.), di Chianale (Vigna Taglianti, com. pers.) e del Vallone di Soustra. Tale morfologia risulta poi costante nei numerosi esemplari provenienti dalle valli a Nord del Monviso.

\section{ECOLOGIA}

Lungo il versante francese delle Alpi Occidentali, $P$. honnoratii risulta essere molto più comune $\mathrm{e}$ diffusa (Pupier com. pers., 2012) rispetto al versante italiano e presenta un excursus altitudinale assai più ampio: la si rinviene, infatti, anche a quote relativamente basse in molte località delle Prealpi di Vaucluse, del Delfinato e del Vercors (v. Catalogo topografico e cfr. Jeannel, 1942). Al Mont Ventoux, ad esempio, popola formazioni miste di latifoglie in cui predomina il faggio a quote intorno a $1350-1500 \mathrm{~m}$, su suoli calcarei molto fessurati e su entrambi i versanti (Casale, com. pers., 2011).

Sul versante italiano, invece, la specie limita la sua presenza quasi esclusivamente alla fascia alpina. La maggior parte delle segnalazioni, infatti, si riferisce a stazioni situate sopra i 1800-2000 m (2700 $\mathrm{m}$ è la quota massima registrata), mentre quelle di vecchia data relative a località situate ad altitudini inferiori ("Coazze", "Balme", ecc.) prive di quota potrebbero riguardare individui fluitati o, comunque, sono da intendere riferite ai monti circostanti. Inoltre, nelle valli piemontesi, la fenologia della specie (come si evince anche dalle date di cattura) tende essere precoce e molto breve (soprattutto su terreni molto drenati). Di conseguenza gli esemplari rinvenuti con le ricerche a vista - con l'eccezione di qualche località della parte centrale del suo areale (v. oltre) - sono quasi sempre poco numerosi, tanto che buona parte delle stazioni note sono state individuate solo grazie a reperti casuali (v. Catalogo topografico), spesso non più verificatisi in anni successivi. Tutto ciò ha senza dubbio contribuito a creare per $P$. honnoratii una fama di specie rara che è in gran parte immeritata, come in effetti sembra dimostrare l'elevato numero di esemplari censito nel Parco OrsieraRocciavrè (cfr. Allegro \& Viterbi, 2010) con l'impiego di trappole a caduta (pitfall traps).

Già Stierlin (1881), segnalando di aver rinvenuto i due individui della serie tipica di sellae (Val Pesio) in prossimità della neve, fece intuire che sul versante italiano la specie è tendenzialmente criofila e perinivale (su quello francese, come già evidenziato, è anche silvicola). A causa di tale praeferendum igrotermico, essa tende a concentrarsi lungo i canaloni e nelle conche dove si accumulano regolarmente consistenti masse nevose di valanga che, con la graduale fusione, ne prolungano la fenologia durante l'estate. È in tali ambienti che, in alcune stazioni piemontesi, essa si rinviene relativamente più numerosa, anche se, indubbiamente, la sua maggiore o minore abbondanza è fortemente influenzata dalle caratteristiche del substrato (v. oltre).

La specie in oggetto è prevalentemente legata a sfasciumi minuti (ghiaioni e substrati ricchi di ciottoli e pietrame) in gran parte denudati (in quanto mobili e regolarmente rimaneggiati dalle valanghe) o appena colonizzati da chiazze di vegetazione pioniera specializzata (Fig. 4), habitat del tutto simile a quello spesso frequentato da altre specie congeneri quali Pterostichus (Parapterostichus) schaschli (Marseul, 1880) nelle Alpi Orientali (Brandmayr \& Zetto Brandmayr, 1988; Brandmayr \& Pizzolotto, 1989), Pterostichus (Platypterus) lombardus K. Daniel, 1901 (Bisio, osservazioni personali) e Pterostichus (Pterostichus) focarilei Casale \& Giachino, 1985 (Casale \& Giachino, 1985). Sembra prediligere i suoli di matrice carbonatica, come del resto dimostra anche l'ampia diffusione sui massicci calcarei francesi: a tale proposito cfr. le cartine della 〈géologie simplifiée〉 e di di- 


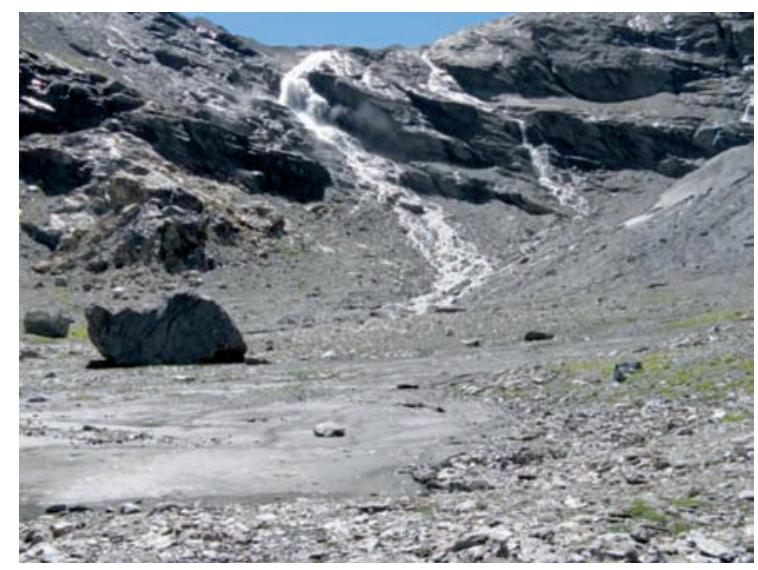

Fig. 4. Rifugio Bezzi (Valgrisenche): nevai su suolo fluvio-glaciale di matrice carbonatica (calcescisti), tipico habitat di P. honnoratii (fotografia di Thomas Götz).

stribuzione della specie in Coulon et al. (2000). Infatti, pur non essendo esclusiva di tali suoli, su substrati silicei o sembra del tutto assente (è il caso, ad esempio, del Massiccio dell'Argentera), o le sue popolazioni paiono molto localizzate e di modesta consistenza (nella catena del Monviso e lungo i contrafforti del Gran Paradiso). La scarsa capacità di ritenzione idrica della maggior parte dei substrati che la specie frequenta contribuisce ad accentuarne notevolmente la contrazione della fenologia, facendola apparire molto sporadica: è il caso dei massicci calcarei e dolomitici delle Alpi Liguri, delle Marittime e delle Cozie meridionali, dove il numero di stazioni note è in quasi tutte le valli piuttosto ridotto (fa eccezione la Valle Stura di Demonte, unicamente per merito della frequentazione pluriennale da parte di Vigna Taglianti). Soltanto nella parte centrale dell'areale (Val Varaita, Val Pellice, Val Chisone e Val di Susa), in corrispondenza degli ampi affioramenti di calcescisti, la presenza di suoli meno drenati consente un prolungamento della fenologia della specie (che qui si rinviene soprattutto sotto sassi scistosi parzialmente interrati), favorendo una maggior quantità di reperti. Qui il numero di stazioni note è maggiore e in alcune di esse è possibile sia osservarne regolarmente popolazioni relativamente numerose al primo fondere delle nevi, sia imbattersi in individui della specie anche in estate avanzata (soprattutto se la stagione è caratterizzata da un elevato tasso di precipitazioni): a tale proposito, oltre a quelle ormai classiche (il Colle delle Finestre e il Vallone dell'Albergian), sono da segnalare il Colle dell'Urina in Val Pellice, il Col Basset in Val Chisone e il Monte Niblé in Val di Susa. Nelle Alpi Graie la specie sembra invece oggettivamente più rara anche in corrispondenza del litotipo sopra citato che affiora, nelle Valli di Lanzo, lungo i contrafforti orientali del Rocciamelone e sui monti sovrastanti il Pian della Mussa: di queste valli, nonostante le ricerche pluriennali effettuate dallo scrivente in ambienti apparentemente favorevoli alla specie, si conoscono soltanto pochissimi esemplari (v. Catalogo topografico).

\section{RINGRAZIAMENTI}

Desidero ringraziare tutti gli amici e colleghi che, in vario modo, mi hanno aiutato a portare a termine il presente lavoro. In particolare, il Prof. René Pupier che, comunicandomi una notevole quantità di dati corologici relativi al territorio francese, mi ha di fatto fornito un quadro esaustivo della distribuzione di P. honnoratii in tale territorio; il Dr. Gianni Allegro per la comunicazione di dati corologici e per l'esecuzione delle fotografie dei pronoti; il Dr. Thomas Götz per la comunicazione dell'interessantissimo dato relativo alla Valgrisenche e per aver concesso l'uso della fotografia del biotopo di rinvenimento; il Dr. Gabriele Franzini per la comunicazione dell'altrettanto interessante dato relativo alla Valle di La Thuile; il Dr. Pier Mauro Giachino e il Prof. Augusto Vigna Taglianti per la comunicazione di numerosi dati corologici. Infine, desidero ancora ringraziare il Prof. Achille Casale per i preziosi suggerimenti, per le informazioni ecologiche relative al Mont Ventoux e per la revisione del manoscritto. 


\section{BIBLIOGRAFIA}

AA.VV., 2007 - ATBI + M: All Taxa Biodiversity Inventories + Monitoring in the Mercantour/Alpi Marittime natural Parks. http://atbi.eu/mercantour-marittime/?q=node/1.

Allegro G., 2000 - Catalogo della collezione Orlando Cantamessa: I. Coleoptera Cicindelidae e Carabidae. Rivista Piemontese di Storia Naturale, 21: 153-192.

Allegro G., Bisio L. \& Negro M., 2011 - I Carabidi di Val Veny e Val Ferret (Coleoptera Carabidae) (Valle d'Aosta, Italia). Bollettino della Società entomologica italiana, 143 (2): 59-76.

Allegro G. \& Chiarabaglio P., 2008 - I Carabidi del Parco naturale del Mont Avic (Valle d'Aosta) (Coleoptera, Carabidae). Revue Valdôtaine d'Histoire Naturelle, 61-62 (2007-2008): 179-188.

Allegro G. \& Viterbi R., 2009 - Note faunistiche ed ecologiche sui carabidi del Parco Nazionale del Gran Paradiso (Coleoptera Carabidae). Rivista Piemontese di Storia Naturale, 30: 129-147.

Allegro G. \& Viterbi R., 2010 - Contributo alla conoscenza faunistica ed ecologica dei Carabidi del Parco Naturale Orsiera Rocciavrè e della Riserva di Foresto (Coleoptera, Carabidae). Rivista Piemontese di Storia Naturale, 31: 187-212.

Bisio L., 1995 - Contributo alla conoscenza di alcuni Pterostichus orofili del Piemonte (Coleoptera Carabidae). Rivista Piemontese di Storia Naturale, 15 (1994): 67-98.

Bisıo L., 2003 - La carabidofauna della Val Soana (Alpi Graie) (Coleoptera Carabidae). Rivista Piemontese di Storia Naturale, 24: $239-288$.

Bisio L., 2004 - Note sui Coleotteri Carabidi dell'alta e media Val Pellice (Alpi Cozie) e osservazioni sulla carabidofauna della Conca del Prà (alta Val Pellice) (Coleoptera Carabidae). Rivista Piemontese di Storia Naturale, 25: 283-329.

Bisıo L., 2007 - I Coleotteri Carabidi della Val Germanasca (Coleoptera Carabidae). Rivista Piemontese di Storia Naturale, 28: $195-245$.

Bisio L., 2010 - I Coleotteri Carabidi della Valle Po (Alpi Cozie) (Coleoptera Carabidae). Rivista Piemontese di Storia Naturale, 31: 139-186.

Bisıo L., 2012 - I Coleotteri Carabidi della Val Chiusella (Alpi Graie) (Coleoptera Carabidae). Rivista Piemontese di Storia Naturale, 33 .

Bisio L. \& Giuntelli P., 2008 - I Coleotteri Carabidi della Val Varaita (Coleoptera Carabidae). Rivista Piemontese di Storia Naturale, 29: 225-278.

Bisio L. \& Giuntelli P., 2011 - I Coleotteri Carabidi della Val Maira (Alpi Cozie) (Coleoptera Carabidae). Rivista Piemontese di Storia Naturale, 32: 173-226.

Brandmayr, P. \& Zetto Brandmayr T., 1988 - Comunità a Coleotteri Carabidi delle Dolomiti Sudorientali e delle Prealpi carniche (con la collaborazione di Roberto Pizzolotto). Studi Trentini di Scienze Naturali, Acta Biologica, 64 Suppl.: 125-250.

Brandmayr P. \& Pizzolotto R., 1989 - Aspetti zoocenotici e biogeografici dei popolamenti a Coleotteri Carabidi nella fascia alpina delle Vette di Feltre (Belluno). Biogeographia (NS), 13: 713-743.

CAillol H., 1908 - Catalogue des Coléoptères de Provence, 1re partie, Société des Sciences Naturelles de Provence, Marseille, $521 \mathrm{pp}$.

Casale A. \& Giachino P.M., 1985 - Un nuovo Pterostichus dell'Appennino Lucano (Coleoptera Carabidae). Bollettino del Museo Regionale di Scienze Naturali Torino, 3 (2):427-436.

Casale A. \& Vigna Taglianti A., 1993 - I Coleotteri Carabidi delle Alpi occidentali e centro-occidentali (Coleoptera Carabidae). Biogeographia, Lavori Società Italiana di Biogeografia, (n. s.), 16 (1992): 331-399.

Casale A., Vigna Taglianti A., Brandmayr P. \& Colombetta G. 2006 - Insecta Coleoptera Carabidae (Carabini, Cychrini, Trechini, Abacetini, Stomini, Pterostichini). In: Ruffo S., Stoch F. (eds.), Ckmap (Checklist and distribution of the Italian fauna). Memorie del Museo Storia Naturale Verona, 2. serie, Sezione Scienze della vita, 17: 159-164, with data on CD-Rom.

Coulon J., Marchal P., Pupier R., Richoux P., Allemand R., Genest L. C., Clary J., 2000 - Coléoptères de Rhône-Alpes, Carabiques et Cicindèles. Société linnéenne de Lyon, 193 pp.

Coulon J., Pupier R., QuÉinnec É., Ollivier É. \& Richoux P., 2011 - Coléoptères Carabiques. Compléments aux deux volumes de René Jeannel, mises à jour, correction et répertoire. Faune de France 95 (vol. 2): 372-685.

DeJean P., 1828 - Species général des Coléoptères de la collection de M. le Comte Dejean. Tome troisième. Paris, MéquignonMarvis, VII + 556 pp. 
FOCARILE A., 1974 - Aspetti zoogeografici del popolamento di coleotteri (Insecta) nella Valle d'Aosta. Revue Valdôtaine d'Histoire Naturelle, 28: 5-53.

FOCARILE A., 1987 - Ecologie et Biogeographie des Coléoptères de haute altitude en Vallée d'Aoste. Regione Autonoma Valle d'Aosta. Assessorato Agricoltura, Foreste e Ambiente naturale, 167 pp., 72 carte, 4 tavv.

JEAnNEL R., 1941 - Coléoptères Carabiques I (Faune de France, vol. 39). P. Lechevalier (Paris), 1-571.

JEAnNEl R., 1942 - Coléoptères Carabiques II (Faune de France, vol. 40). P. Lechevalier (Paris), 573-1173.

Löbl I. \& Smetana A. (eds.), 2003 - Catalogue of Palaearctic Coleoptera. I. Archostemata-Myxophaga-Adephaga. Apollo Books, Stenstrup, 819 pp.

LORENZ W. 2005 - Systematic list of extant ground beetles of the world (Insecta Coleoptera "Geadephaga": Trachypachidae and Carabidae incl. Paussinae, Cicindelinae, Rhysodinae). Second Edition. Tutzing, Germany, iv + 530 pp.

Magistretti M., 1965 - Fauna d'Italia, VIII. Coleoptera: Cicindelidae, Carabidae. Catalogo topografico. Calderini, Bologna, $512 \mathrm{pp}$.

Magistretti M., 1968 - Catalogo topografico dei Coleptera Cicindelidae e Carabidae d'Italia. I Supplemento. Memorie della Società entomologica italiana, 47: 177 -217.

PorTA A., 1923 - Fauna Coleopterorum Italica. I. Adephaga. Piacenza, 285 pp.

PuPIER R., 2007 - Désignation d'un lectotypus de Pterostichus honnoratii sellae Stierlin, 1881 (Coleoptera, Carabidae, Pterostichini). Nouvelle Revue d'Entomologie, 24/1: 82-83.

Sainte-Claire Deville J., 1902 - Étude sur divers Platysma des alpes occidentales. Annales de la Société entomologique de France, 71: 588-619.

SChATZMAYR A., 1930 - I Pterostichus italiani. Memorie della Società entomologica italiana, 8 (1929): 145-339.

SCHATZMAYR, A. (1942-43) - Bestimmunsgtabellen der europäischen und nordafrikanischen Pterostichus- und TapinopterusArten. Koleopterologische Rundschau, 27: 1-144.

STIERLIN W.G., 1881 - Ein neuer Pterostichus aus den Seealpen. Mittheilungen der Schweizerischen Entomologischen Gesellschaft, 6 (4): 142.

Vigna TAglianti A., 2004 - Fauna Europaea: Carabidae. In: Audisio P. (ed.), 2004. Coleoptera 2. Fauna Europaea version 1.1, http://www.faunaeur.org.

Vigna Taglianti A., 2005 - Checklist e corotipi delle specie di Carabidae della fauna italiana. Appendice B. pp. 186-225. In: P. Brandmayr, T. Zetto \& R. Pizzolotto (eds.), I Coleotteri Carabidi per la valutazione ambientale e la conservazione della biodiversità. Manuale operativo. APAT, Manuali e Linee Guida, 34: 240 pp.

WINKLER A., 1924-1932 - Catalogus Coleopterorum regionis palaearcticae. A. Winkler ed., Wien, 1698 pp.

Indirizzo dell'autore:

L. Bisio, via Galilei 4, 10082 Cuorgné (TO). E-mail: luigibisio@virgilio.it 